\title{
Requirement of the Poly(A) Tail in Coronavirus Genome Replication
}

\author{
JEANNIE F. SPAGNOLO AND BRENDA G. HOGUE \\ Department of Molecular Virology and Microbiology, Baylor College of Medicine, Houston, \\ Texas
}

\section{INTRODUCTION}

The $3^{\prime}$ poly(A) tail plays an important, but as yet undefined role in coronavirus genome replication. To further examine the requirement for the coronavirus poly(A) tail, we created truncated poly(A) mutant defective interfering (DI) RNAs and observed the effects on replication. Bovine coronavirus (BCV) and mouse hepatitis coronavirus A59 (MHV-A59) DI RNAs with tails of 5 or 10 A residues were replicated, albeit at delayed kinetics as compared to DI RNAs with wild type tail lengths ( $>50 \mathrm{~A}$ residues). A BCV DI RNA lacking a poly(A) tail was unable to replicate; however, a MHV DI lacking a tail did replicate following multiple virus passages. Poly(A) tail extension/repair was concurrent with robust replication of the tail mutants. Binding of the host factor poly(A)- binding protein (PABP) appeared to correlate with the ability of DI RNAs to be replicated. Poly(A) tail mutants that were compromised for replication, or that were unable to replicate at all exhibited less in vitro PABP interaction. The data support the importance of the poly(A) tail in coronavirus replication and further delineate the minimal requirements for viral genome propagation. 


\section{BACKGROUND}

The coronavirus $5^{\prime}$ and $3^{\prime}$ untranslated regions (UTRs) are necessary for genome replication. The $3^{\prime}$ terminal 55 nucleotides of the $3^{\prime}$ UTR and the poly(A) tail comprise the cis-acting signal for negative strand synthesis (Lin et al., 1994). Defective genome positive strand synthesis requires UTRs from both the 5' and 3' ends of the genome (Kim et al., 1993; Lin and Lai, 1993). The precise roles of these UTRs during replication are unknown, but it is likely that they function to recruit viral factors and possibly cellular proteins for formation of the viral replication complex.

The poly(A) tail has been shown to be important for replication of several positive-strand viruses including polio (Sarnow, 1989; Spector and Baltimore, 1974), EMCV (Cui et al., 1993), and Sindbis (Hill et al., 1997). MHV RNAs contain poly(A) tails of approximately 100-130 nt (Lai et al., 1981). The mechanism by which the tail is added to coronavirus RNA is not known, though the presence of a 9-26 nt poly(U) tract at the $5^{\prime}$ end of $\mathrm{BCV}$ negative-strand RNAs alludes to the likelihood that negative-strand synthesis initiates within the poly(A) tail (Hofmann and Brain, 1991). It remains to be determined how the tail is extended beyond the templated 9-26 nt. Irrespective of the mechanism of tail addition, the presence of a poly(A) tail of undetermined length is required for negative strand synthesis, the initial step of genome replication (Lin et al., 1994).

To further investigate the requirement for the poly(A) tail in coronavirus replication, we sought to determine the effect of poly(A) tail truncation on DI RNA replication. DI replication was monitored over several virus passages and visualized by Northern blotting of total intracellular RNA. Repair of truncated poly(A) tails over the course of the experiment was examined by RT-PCR. We recently showed that PABP interacts with the poly(A) tail on group II coronavirus RNA (Spagnolo and Hogue, 2000). To determine whether binding of the host factor PABP correlated with the replication phenotypes of the mutants, a streptavidin-capture assay was developed to measure interactions with PABP in vitro.

\section{MATERIALS AND METHODS}

Defective genome replication, PABP-3' UTR binding, and RT$\mathrm{PRC} /$ poly(A) tail repair assays were all described in detail previously (Spagnolo and Hogue, 2000). 


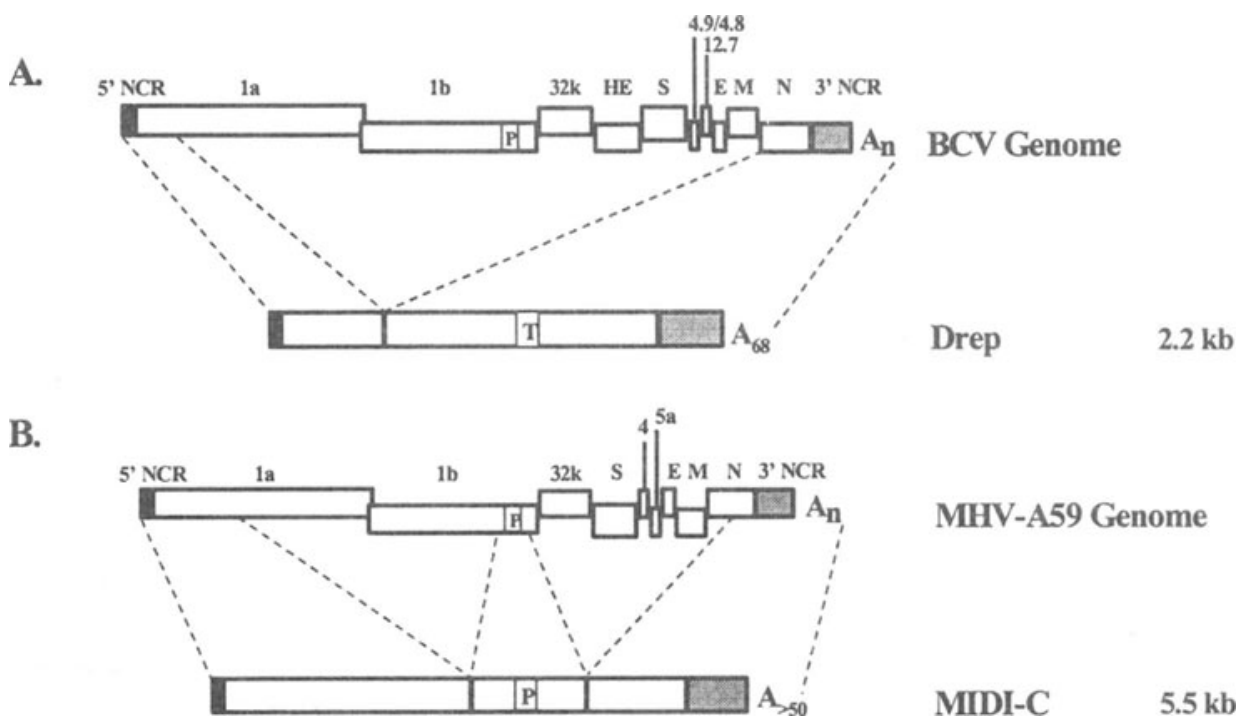

Figure 1. Schematic of BCV and MHV-A59 wt and defective genomes. The genes encoded by the open reading frames (white boxes) are labeled. The leader and 5' UTR are shown in black and the 3' UTR is shown crosshatched. Sequences found in BCV Drep (Chang et al., 1994) and MHV MIDI-C (de Groot et al., 1992) defective genomes are aligned with the position of origin in the parental genome and are indicated by dotted lines. Drep contains a 30 nt TGEV (T) reporter sequence. "P" indicates the position of the MHV and BCV packaging signals.

To determine the effect of poly(A) tail truncation on DI RNA replication, Drep and MIDI-C RNAs were constructed that contained poly(A) tails of $0 / 1,5$, or 10 A residues. In vitro transcribed, capped RNAs were transfected into mock- and BCV-infected HCT cells or MHV-infected $17 \mathrm{Cll}$ cells. Passage of virus supernatants onto new cell monolayers was performed at 24 $\mathrm{h}$ timepoints for $\mathrm{BCV}$, and $12 \mathrm{~h}$ timepoints for MHV. DI replication was monitored over four virus passages by Northern blot analysis. Little to none of the transfected DI RNAs persisted in mock- infected HCT (Fig. 2A lanes 2-3) or 17Cll cells (Fig. 2B lanes 3-4). All Drep RNAs were present at $24 \mathrm{~h}$ for $\mathrm{BCV}$ (Fig. 2A lane 5); however, only Drep $A_{5}, A_{10}$, and $A_{w t}$ were replicated upon virus passage (Fig. 2A lower panels, lanes 6-9). Drep $A_{1}$ was not replicated, or underwent replication so inefficiently that accumulation of this RNA was not detected by Northern blotting (Fig. 2A, upper panel, lanes 6-9). As was seen for Drep, MIDI-C $A_{5}, A_{10}$, and $A_{w t}$ were clearly replicated and amplified upon virus passage (Fig. 2B, lower panels, lanes 7-10). Interestingly, MIDI-C $\mathrm{A}_{0}$ accumulation was also observed by P3 or P4 (Fig 2B, upper panel, lanes 7-10). 


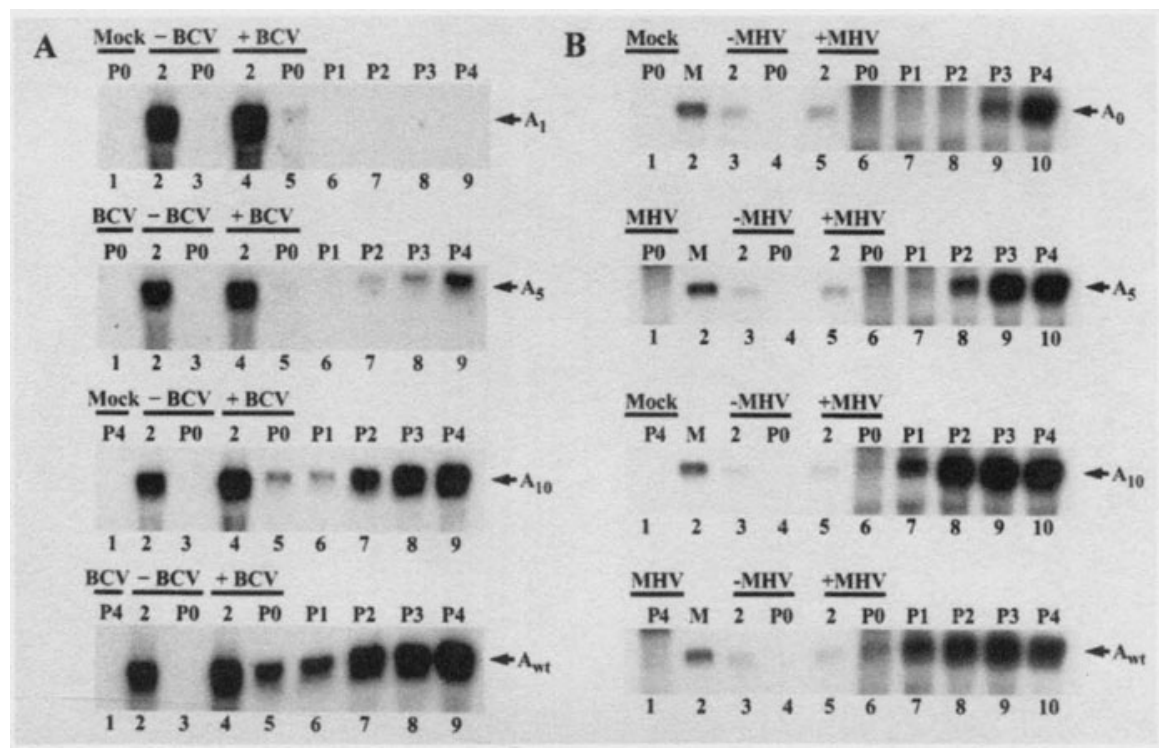

Figure 2. Replication of $\mathrm{BCV}$ and $\mathrm{MHV}$ defective genomes containing varying lengths of poly(A) tail. Mock- and BCV-infected HCT cells (A) or MHV-infected 17Cll cells (B) were transfected with Drep (A) or MIDI-C (B) RNAs. Total intracellular RNA was extracted, resolved on denaturing agarose gels, and vacuum blotted onto nylon membranes. Blots were probed with a 5'-end-labeled oligonucleotide complementary to the TGEV reporter sequence in Drep (A) or an MHV N riboprobe (B). P1-P4 indicates virus passage numbers. Virus passages were performed at $24 \mathrm{~h}$ intervals for $\mathrm{BCV}$ and $12 \mathrm{~h}$ for MHV. M denotes marker RNA (B).

To rule out any possibility that the observed differences in replication could be due to differences in RNA decay rates, stability of the transfected DI RNAs was monitored over a $12-24 \mathrm{~h}$ period following transfection by Northern blotting. All RNAs were comparable in stability and exhibited similar decay rates (Spagnolo and Hogue, 2000). Drep RNAs had a half-life of $10 \mathrm{~h}$, and MIDI-C RNAs had a half-life of approximately $5.5 \mathrm{~h}$. Quantitation of this data was performed by phosphorimaging. It therefore did not appear that any striking difference in RNA stability could account for the differences in replication competency of the mutant DI RNAs.

To determine whether binding of the host factor PABP better correlated with DI replication phenotypes, an assay was developed to detect PABP interactions with the BCV 3' or MHV3' UTRs containing various lengths of poly(A) tail. ${ }^{35} \mathrm{~S}$ labeled PABP, or luciferase as a negative control, was incubated with biotinylated probes in the presence of nonspecific competitors. Immobilized streptavidin was added to recover the biotinylated RNA-protein complexes. Half of each sample was analyzed for recovery of 
radiolabeled protein by SDS-PAGE. The other half was monitored for comparable recovery of all biotinylated RNAs. No binding above background was detected between luciferase and any of the 3' UTR poly(A) tail RNAs. Binding of PABP to BCV 3' UTR $\mathrm{A}_{5}, \mathrm{~A}_{10}$, and $\mathrm{A}_{\mathrm{wt}}$, and to MHV 3' UTR $A_{10}$ and $A_{w t}$ was detected (Fig. 3). Folding of the 3' UTR $A_{5}$ may account for lack of PABP binding. This data demonstrates that binding of PABP to 3' UTR RNAs correlates with the ability of the DI poly(A) tail mutants to be replicated.
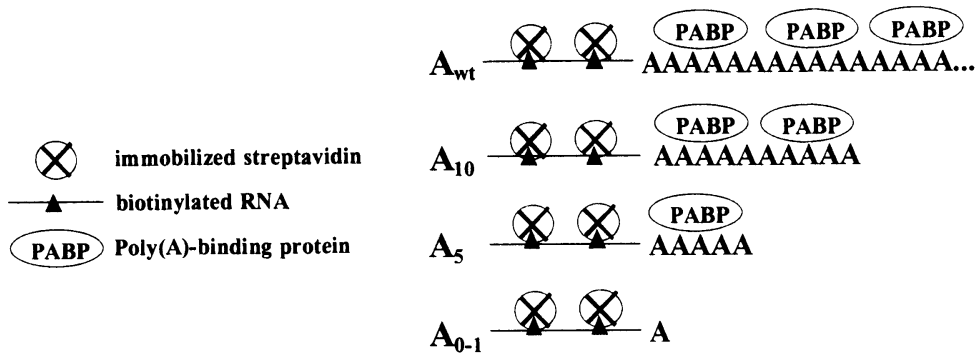

\begin{tabular}{lc}
$\begin{array}{c}\text { Fold PABP binding } \\
\text { over background } \\
\text { BCV3, }\end{array}$ & MHV3, \\
\hline 31 & 81 \\
24 & 48 \\
15 & 2 \\
1.5 & 2
\end{tabular}

Figure 3. Schematic representation of in vitro binding of PABP to coronavirus 3' UTR RNAs. In vitro translated PABP was incubated with biotinylated BCV3'UTR or MHV3'UTR RNAs containing poly(A) tails of $0-1,5,10$, or wt $(>50)$ A-residues. Immobilized streptavidin was added to recover biotinylated RNA complexes, and samples were washed to remove any unbound RNA or protein. Samples were analyzed on $8 \%$ SDS-PAGE. PABP binding was quantitated by phosphorimaging.

The observation that MIDI-C $\mathrm{A}_{0}$ was competent for replication by $\mathrm{P} 3$ or $\mathrm{P} 4$ led us to speculate that MIDI-C $\mathrm{A}_{0}$ had undergone poly(A) tail repair over the duration of the experiment and thus became a suitable template for replication. To determine whether this was indeed the case, an RT-PCR assay was utilized. DI RNAs containing poly(A) tails of $>10$ A residues produce an $850 \mathrm{bp}$ PCR product, whereas RNAs with tails $\leq 10 \mathrm{~A}$ residues are not amplified. Intracellular RNAs from the experiment shown in Fig. 2 were subject to RT-PCR analysis. Table 1 represents the results of these experiments. "+" denotes the appearance of the predicted $850 \mathrm{bp} \mathrm{RT-PCR}$ product, "-" indicates failure to amplify this product. MIDI-C $A_{w t}$, whose poly(A) tail is well over $10 \mathrm{~A}$ residues, yielded the $850 \mathrm{bp}$ product from all virus passages, as expected. RT-PCR products for the poly(A) tail mutants were observed simultaneously with the appearance of robust replication of the DI RNAs (compare Table 1 with Fig. 2). These data demonstrate that in vivo, MIDI-C $\mathrm{A}_{0}, \mathrm{~A}_{5}$, and 10 underwent poly(A) tail repair and were replicated. 
Table 1. Appearance of RT-PCR product indicating poly(A) repair

\begin{tabular}{llllll}
\hline Transcript & P0 & P1 & P2 & P3 & P4 \\
\hline MIDI-C A $A_{0}$ & -- & -- & -- & ++ & +++ \\
MIDI-C A & -- & $-/+$ & ++ & +++ & +++ \\
MIDI-C A $A_{10}$ & -- & ++ & +++ & +++ & +++ \\
MIDI-C $A_{w t}$ & ++ & ++ & +++ & +++ & +++ \\
\hline
\end{tabular}

\section{CONCLUSION}

Our understanding of the molecular interactions required for coronavirus genome replication remains largely incomplete. In an effort to further elucidate the requirements for this process, we have begun to more closely examine the coronavirus $3^{\prime}$ UTR and the poly(A) tail. In this report, we confirmed previous results indicating that the poly(A) tail is a cis-acting signal for coronavirus replication, and further showed that a poly(A) tail of 5 A residues is sufficient for initial DI replication. In vitro binding of the host factor PABP appeared to correlate with the ability of DI RNAs to be replicated.

Our data showed that poly(A) tail truncation compromised the ability of DI RNAs to be replicated, as DIs with truncated tails did replicate, but exhibited a decrease in the overall amount of replication. Poly(A) tail deletion resulted in failure of $B C V$ Drep $A_{1}$ RNA to be replicated, whereas MHV MIDI-C $A_{0}$ was able to overcome any block to replication by passage three or four. RNA stability did not account for the differences in replication. It appears that $\mathrm{BCV}$ and MHV differ in their ability to deal with poly(A)-lacking DIs. MIDI-C $A_{0}$ underwent poly(A) tail extension during the course of the replication experiment and regained replication competency, whereas Drep $A_{1}$ became extinct. If repair occurs by a virusspecific mechanism, then MHV is able to repair MIDI-C $A_{0}$ 's defect in the four virus passages we have examined, whereas $\mathrm{BCV}$ is unable to do so for Drep $A_{1}$. We are currently trying to determine how repair occurs. It is likely due to recombination with helper virus, and if this is the case, would suggest that MHV may undergo recombination more readily than BCV.

Binding of the host factor PABP appears to correlate with the ability of the mutant DI RNAs to be replicated. What roles may PABP and the coronavirus poly(A) tail be playing during coronavirus replication? $\mathrm{PABP}$ and the poly(A) tail likely exert an initial effect on genome translation immediately after infection. PABP interactions with the coronavirus poly(A) tail and eIF4G, which is presumably bound to the coronavirus genome $5^{\prime}$ cap, likely mediate $5^{\prime}-3^{\prime}$ end interactions and may facilitate genome translation. As translation appears to be required for efficient genome replication (Chang and Brian, 1996; van der Most et al., 1995), 
poly(A) lacking DIs may be compromised in translation efficiency, and thereby do not replicate as well, or at all.

The coronavirus poly(A) tail likely plays an additional, direct role in genome replication by serving as part of the negative strand promoter. $\mathrm{BCV}$ negative strand RNA contains a 9-26 poly(U) tract, suggesting that negative strand RNA synthesis likely initiates within the poly(A) tail (Hofmann and Brain, 1991). DIs with shorter or nonexistent tails may therefore not be as efficient at or capable of being templates for negative strand RNA synthesis. Finally, initial interactions mediated in part by PABP that presumably juxtapose the $5^{\prime}$ and $3^{\prime}$ ends of coronavirus RNA may be important for building the replicase complex and may explain the requirement of sequences from both $5^{\prime}$ and $3^{\prime}$ ends of the RNA for positive strand synthesis.

\section{ACKNOWLEDGMENTS}

This work was supported by Public Health Service NIH grant AI33500 to B.G.H. from the National Institute of Allergy and Infectious Diseases. J.F.S. was supported in part by training grant AI07471 from the National Institutes of Health.

\section{REFERENCES}

Chang, R.Y. and Brian, D.A.(1996). cis Requirement for N-specific protein sequence in bovine coronavirus defective interfering RNA replication. J Virol 70, 2201-2207.

Chang, R.Y., Hofmann, M.A., Sethna, P.B., and Brian, D.A.(1994). A cis-acting function for the coronavirus leader in defective interfering RNA replication. J Virol 68, 8223-8231.

Cui, T., Sankar, S., and Porter, A.G.(1993). Binding of encephalomyocarditis virus RNA polymerase to the 3'-noncoding region of the viral RNA is specific and requires the 3'poly(A) tail. J Biol Chem 268, 26093-26098.

de Groot, R.J., van der Most, R.G., and Spaan, W.J.(1992). The fitness of defective interfering murine coronavirus DI-a and its derivatives is decreased by nonsense and frameshift mutations. J Virol 66, 5898-5905.

Hill, K.R., Hajjou, M., Hu, J.Y., and Raju, R.(1997). RNA-RNA recombination in Sindbis virus: roles of the 3 ' conserved motif, poly(A) tail, and nonviral sequences of template RNAs in polymerase recognition and template switching. J Virol 71, 2693-2704.

Hofmann, M.A. and Brian, D.A.(1991). The 5' end of coronavirus minus-strand RNAs contains a short poly $(\mathrm{U})$ tract. J Virol 65, 6331-6333.

Kim, Y.N., Jeong, Y.S., and Makino, S.(1993). Analysis of cis-acting sequences essential for coronavirus defective interfering RNA replication. Virology 197, 53-63.

Lai, M.M., Brayton, P.R., Armen, R.C., Patton, C.D., Pugh, C., and Stohlman, S.A.(1981). Mouse hepatitis virus A59: mRNA structure and genetic localization of the sequence divergence from hepatotropic strain MHV-3. J Virol 39, 823-834. 
Lin, Y.J. and Lai, M.M.(1993). Deletion mapping of a mouse hepatitis virus defective interfering RNA reveals the requirement of an internal and discontiguous sequence for replication. J Virol 67, 6110-6118.

Lin, Y.J., Liao, C.L., and Lai, M.M.(1994). Identification of the cis-acting signal for minusstrand RNA synthesis of a murine coronavirus: implications for the role of minus-strand RNA in RNA replication and transcription. J Virol 68, 8131-8140.

Sarnow, P.(1989). Role of 3 '-end sequences in infectivity of poliovirus transcripts made in vitro. J Virol 63, 467-470.

Spagnolo, J.F. and Hogue, B.G.(2000). Host protein interactions with the $3^{\prime}$ end of bovine coronavirus RNA and the requirement of the Poly(A) tail for coronavirus defective genome replication. J Virol 74, 5053-5065.

Spector, D.H. and Baltimore, D.(1974). Requirement of 3'-terminal poly(adenylic acid) for the infectivity of poliovirus RNA. Proc Natl Acad Sci U S A 71, 2983-2987.

van der Most, R.G., Luytjes, W., Rutjes, S., and Spaan, W.J.(1995). Translation but not the encoded sequence is essential for the efficient propagation of the defective interfering RNAs of the coronavirus mouse hepatitis virus. J Virol 69, 3744-3751. 